\title{
Enhanced expression of centromere protein F predicts clinical progression and prognosis in patients with prostate cancer
}

\author{
YANG-JIA ZHUO $^{1-3^{*}}$, MING XI $^{1 *}$, YUE-PING WAN ${ }^{1 *}$, WEI HUA $^{1}$, YUAN-LING LIU $^{1}$, SONG WAN $^{1}$, \\ YU-LIN ZHOU ${ }^{1}$, HONG-WEI LUO ${ }^{2}$, SHU-LIN WU ${ }^{4,5}$, WEI-DE ZHONG ${ }^{1-3}$ and CHIN-LEE WU ${ }^{4,5}$ \\ ${ }^{1}$ Department of Urology, Huadu District People's Hospital, Southern Medical University, Guangzhou, \\ Guangdong 510800; ${ }^{2}$ Guangdong Provincial Institute of Nephrology, Southern Medical University, Guangzhou, \\ Guangdong 510515; ${ }^{3}$ Department of Urology, Guangdong Key Laboratory of Clinical Molecular Medicine and Diagnostics, \\ Guangzhou First People's Hospital, Guangzhou Medical University, Guangzhou, Guangdong 510180, P.R. China; \\ Departments of ${ }^{4}$ Pathology and ${ }^{5}$ Urology, Massachusetts General Hospital \\ and Harvard Medical School, Boston, MA 02114, USA
}

Received October 27, 2014; Accepted January 22, 2015

DOI: $10.3892 / \mathrm{ijmm} .2015 .2086$

\begin{abstract}
Centromere protein F (CENPF) is a protein associated with the centromere-kinetochore complex and chromosomal segregation during mitosis. Previous studies have demonstrated that the upregulation of CENPF may be used as a proliferation marker of malignant cell growth in tumors. The overexpression of CENPF has also been reported to be associated with a poor prognosis in human cancers. However, the clinical significance of CENPF in prostate cancer (PCa) has not yet been fully elucidated. Thus, the aim of the present study was to determine the association of CENPF with tumor progression and prognosis in patients with $\mathrm{PCa}$. The expression of CENPF at the protein level in human PCa and non-cancerous prostate tissues was detected by immunohistochemical analysis, which was further validated using a microarray-based dataset (NCBI GEO accession no: GSE21032) at the mRNA level. Subsequently, the association of CENPF expression with the clinicopathological characteristics of the patients with PCa was statistically analyzed. Immunohistochemistry and dataset analysis revealed that CENPF expression was significantly increased in the PCa tissues compared with the non-cancerous prostate tissues [immunoreactivity score (IRS): PCa, 177.98 \pm 94.096 vs. benign,
\end{abstract}

Correspondence to: Professor Wei-De Zhong, Department of Urology, Guangdong Key Laboratory of Clinical Molecular Medicine and Diagnostics, Guangzhou First People's Hospital, Guangzhou Medical University, 1 Panfu Road, Guangzhou, Guangdong 510180, P.R. China

E-mail: zhongwd2009@live.cn

${ }^{*}$ Contributed equally

Key words: prostate cancer, centromere protein F, clinicopathological characteristic, biochemical recurrence-free survival
$121.30 \pm 89.596, \mathrm{P}<0.001 ; \mathrm{mRNA}$ expression in the dataset: $\mathrm{PCa}, 5.67 \pm 0.47$ vs. benign, $5.40 \pm 0.11 ; \mathrm{P}<0.001]$. Additionally, as revealed by the dataset, the upregulation of CENPF mRNA expression in the PCa tissues significantly correlated with a higher Gleason score (GS, $\mathrm{P}=0.005)$, an advanced pathological stage $(\mathrm{P}=0.008)$, the presence of metastasis $(\mathrm{P}<0.001)$, a shorter overall survival $(\mathrm{P}=0.003)$ and prostate-specific antigen (PSA) failure $(\mathrm{P}<0.001)$. Furthermore, both univariate and multivariate analyses revealed that the upregulation of CENPF was an independent predictor of poor biochemical recurrence (BCR)-free survival ( $\mathrm{P}<0.001$ and $\mathrm{P}=0.012$, respectively). Our data suggest that the increased expression of CENPF plays an important role in the progression of PCa. More importantly, the increased expression of CENPF may efficiently predict poor $\mathrm{BCR}$-free survival in patients with $\mathrm{PCa}$.

\section{Introduction}

Prostate cancer $(\mathrm{PCa})$ is one of the most frequently diagnosed cancers among males, and is the second leading cause of cancerrelated mortality in Western countries. More than 900,000 new cases of PCa are diagnosed worldwide each year (1). In 2014, approximately $24 \%$ of new cancer cases among American males are $\mathrm{PCa}$ (2). The incidence and mortality of PCa in China also appears to be continuously increasing (3). As a clinically heterogeneous multifocal disease, its natural history is highly variable and difficult to predict. The mechanisms influencing the progression and prognosis of PCa are multistep processes, including both genetic insults to epithelial cells and alterations in epithelial-stromal interaction (4). Due to the innovation of surgical techniques and the reduced incidence of surgical complications, many patients with localized $\mathrm{PCa}$ have experienced long-term survival through the intervention of radical prostatectomy (5). However, approximately $20 \%$ of patients with PCa suffer from biochemical recurrence (BCR) following radical prostatectomy or radiation and require further interventions (6). Furthermore, no effective therapeutic treatment is yet available for recurrent or metastatic disease from failed surgery, 
radiation, chemotherapy or hormonal therapy. Clinical variables, such as serum prostate-specific antigen (PSA) levels, Gleason score (GS), margin status and the response to initial treatment, in various combinations, have been used to predict the disease outcome $(7,8)$. However, currently there are no definitive clinical methods for the diagnosis and determination of the disease outcome. Therefore, it is of great importance to identify novel and effective biomarkers involved in the fundamental aspects of tumor biology to provide valuable information for the early diagnosis and tumor progression of $\mathrm{PCa}$.

Although there has been limited success in identifying high-risk susceptibility genes analogous to breast cancer 1 , early onset (BRCA1) or breast cancer 2, early onset (BRCA2) for breast and ovarian cancer, some candidate susceptibility genes which are associated with the initiation and progression of PCa have been identified, such as the amplified genes on chromosomes 1 and X (9).

The cell cycle progression (CCP) score, a novel RNA expression signature derived from $31 \mathrm{CCP}$ genes, has recently been shown to be a strong predictor of clinical outcome in patients with PCa (10-12). Centromere protein F (CENPF), at chromosome 1q41, is one of these 31 genes. It encodes a protein that acts as part of the centromere-kinetochore complex and is a component of the nuclear matrix during the $\mathrm{G} 2$ phase of interphase (13). CENPF is expressed in a cell cycle-dependent manner and is involved in chromosome segregation. CENPF gradually accumulates during the cell cycle until it reaches peak levels in the $\mathrm{G} 2 / \mathrm{M}$ phase and is rapidly degraded upon the completion of mitosis (14). The results of previous studies have demonstrated that CENPF may play a role in the regulation of cell division and may be used as proliferation marker of malignant cell growth in clinical practice due its localizations in the cell cycle (13-16). Accumulating evidence has demonstrated the involvement of CENPF in various types of human cancer, such as breast cancer (17), hepatocellular carcinoma (18), colorectal gastrointestinal stromal tumors (19), nasopharyngeal carcinoma (20), non-Hodgkin's lymphoma (21), salivary gland tumors (22) and neuroblastic tumors (23). The overexpression of CENPF has also been reported to be associated with a poor prognosis in hepatocellular carcinoma, breast cancer, colorectal gastrointestinal stromal tumors and nasopharyngeal carcinoma (17-20). However, the clinical significance of CENPF in PCa has yet not been fully elucidated. Thus, the aim of the present study was to investigate the association of CENPF with tumor progression and prognosis in patients with $\mathrm{PCa}$.

\section{Materials and methods}

Selection of CENPF from the CCP genes. To reliably identify the candidate genes that are associated with tumor progression and prognosis in patients with $\mathrm{PCa}$, we examined the prognostic value of the $31 \mathrm{CCP}$ genes by statistically analyzing the BCR-free survival in a microarray-based dataset (NCBI GEO accession no: GSE21032), contributed by Taylor et al (24) (herein referred to as the Taylor dataset), which is a relatively large and diverse PCa dataset with microarray expression data for microRNAs (miRNAs or miRs) and protein coding genes (mRNAs). From the 218 prostate tumor samples in this dataset, 149 matched normal samples, 12 cell lines and xenografts, only 185 samples, including 150 PCa, 29 adjacent normal tissue and
Table I. Clinical characteristics of all patients.

\begin{tabular}{lcc}
\hline & \multicolumn{2}{c}{ Experiment type } \\
\cline { 2 - 3 } Clinical characteristics & TMA & $\begin{array}{c}\text { Taylor } \\
\text { dataset }\end{array}$ \\
\hline Prostate cancer (cases) & 99 & 150 \\
Mean age (years) & $70.71 \pm 8.00$ & $58.34 \pm 7.07$ \\
$\leq 66$ & 26 & 125 \\
$>66$ & 73 & 25 \\
Serum PSA levels (ng/ml) & & \\
$\quad<4$ & - & 24 \\
$\geq 4$ & - & 123 \\
Gleason score & & \\
$\leq 6$ & 26 & 41 \\
7 & 45 & 76 \\
$\geq 8$ & 28 & 22 \\
Metastasis & 0 & 29 \\
Adjacent benign tissue (cases) & 81 & \\
\hline
\end{tabular}

-, indicates lack of relative information on the patients in our cohort. TMA, tissue microarray (tissues analyzed by immunohistochemistry). Taylor dataset, dataset contributed by Taylor et al (24). All 150 patients in the Taylor dataset were given a follow-up examination ranging from 1 to 175 months (median, 55 months). For the analysis of survival and follow-up, the date of prostatectomy was used as the beginning of the follow-up period. The primary analysis endpoint for the cohort of patients was time to biochemical recurrence. Other analysis endpoint was overall survival. All the patients who succumbed to diseases other than prostate cancer or unexpected events were excluded from the cohort.

6 cell lines, had exon and whole-transcript expression. Thus, we selected these 185 samples for our research.

Patients and tissue samples. For immunohistochemical analysis, a tissue microarray (TMA, $\mathrm{n}=180$ ) including 99 primary PCa tissues and 81 adjacent non-cancerous prostate tissues was obtained from Shanghai Outdo Biotech Co., Ltd., (Shanghai, China; Cat no: HPro-Ade180PG-01), including detailed clinical information. Patients who had been administered chemotherapy or radiotherapy prior to surgery were excluded from this study. In order to investigate the expression of CENPF at the mRNA level and perform survival analysis, the clinical information from the Taylor dataset, including 150 PCa tissues and 29 adjacent non-cancerous prostate tissues was also collected. The detailed information on the clinical characteristics of all the patients in the present study is presented in Table I.

Immunohistochemical analysis. The specimens were fixed in $10 \%$ neutral-buffered formalin and subsequently embedded in paraffin. The paraffin-embedded tissues were cut at $4 \mu \mathrm{m}$ and then deparaffinized with xylene and rehydrated for further peroxidase [3,3'-diaminobenzidine (DAB)] immunohistochemical staining using the Dako EnVision System (Dako Diagnostics AG, Zug, Switzerland). In brief, the tissue 


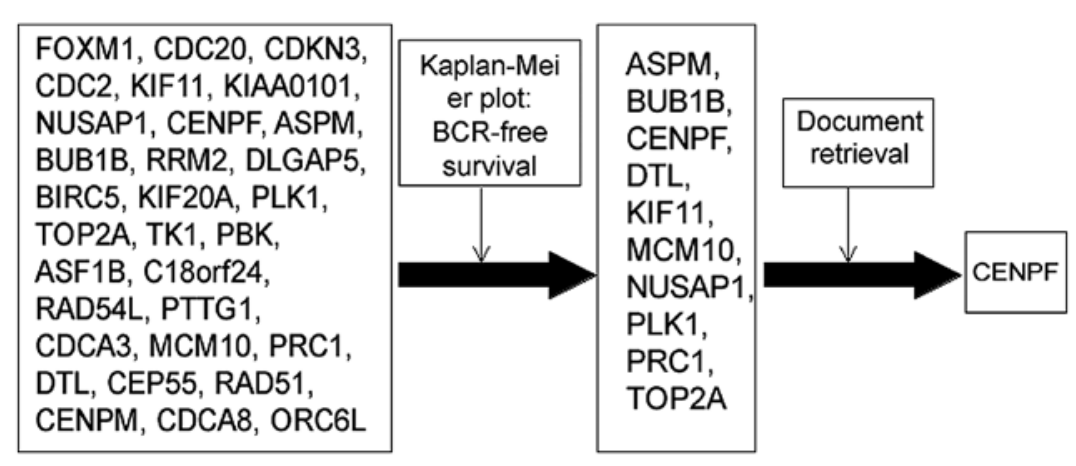

Figure 1. Flow chart showing the selection of centromere protein $\mathrm{F}$ (CENPF).

slides were subjected to proteolytic digestion and blocked with peroxidase, and were then incubated overnight with primary antibody against CENPF (rabbit polyclonal antibody, bs-7839R; Beijing Biosynthesis Biotechnology Co., Ltd., Beijing, China) at a dilution of 1:200 at $4^{\circ} \mathrm{C}$. After washing with PBS, peroxidase-labeled polymer and substrate-chromogen were then employed to visualize the staining of the protein of interest. In each immunohistochemistry run, negative controls were carried out by omitting the primary antibody.

Following counterstaining with hematoxylin, immunostaining was scored by 2 independent experienced pathologists, who were blinded to the clinicopathological data and clinical outcomes of the patients. The scores of the 2 pathologists were compared and any discrepancies in the scores were resolved by the re-examining of the stainings by both pathologists to achieve a consensus score. The immunolabeling of the cancer cells was evaluated. The number of positively stained cells in 10 representative microscopic fields was counted and the percentage of positive cells was also calculated. According to the antibody specification sheet, cytoplasmic staining was regarded as a positive signal. Given the heteogenicity of the staining of the target proteins, tumor specimens were scored in a semi-quantitative manner. Protein levels were determined by the percentage of staining (i.e., from 0 to 100\%) and intensity level of staining [i.e., 0 (negative), 1 (weak), 2 (moderate) and 3 (strong)] in each tumor sample. A final immunoreactivity score (IRS) was obtained by multiplying the percentage of staining and the intensity level for each tumor sample.

Statistical analysis. Statistical analyses were performed by using SPSS version 17.0 software for Windows (SPSS Inc., Chicago, IL, USA). The independent Student's t-test was used to analyze the results and data are expressed as the means \pm SD. The Mann-Whitney test was used for non-normally distributed data. Statistical analysis were performed using Fisher's exact test for any $2 \times 2$ tables, the Pearson $\chi^{2}$ test for non- $2 \times 2$ tables, Kaplan-Meier plots for survival analysis and the Cox proportional hazards regression model for univariate and multivariate survival analyses. Differences were considered statistically significant when the P-value was $<0.05$.

\section{Results}

Selection of CENPF from the CCP genes. Since the expression of the $31 \mathrm{CCP}$ genes is based on the RNA level, it is likely to represent a fundamental aspect and may prove to be useful in determining the clinical outcome of patients with PCa. Thus, we analyzed the $31 \mathrm{CCP}$ genes in the Taylor dataset using Kaplan-Meier plots. The results revealed that the increased expression of 10 genes may correlate with a shorter BCR-free survival. In these 10 genes, CENPF may be used as a proliferation marker of malignant cell growth (Fig. 1). At the same time, it has also been reported to be associated with a poor prognosis in various types of human cancer (17-20).

CENPF protein expression is upregulated in PCa clinical specimens. We first investigated whether the expression of CENPF is associated with clinical specimens of PCa using a TMA (Table I). In this TMA, the expression profile and localization of CENPF in the $99 \mathrm{PCa}$ and 81 adjacent non-cancerous prostate tissues were examined by immunohistochemical analysis (Fig. 2). Immunohistochemical staining revealed that CENPF immunostaining occurred mainly in the cytoplasm in the cells from the PCa tissue; however, weak or moderate staining was observed in the adjacent non-cancerous prostate tissues (Fig. 2A-D). Furthermore, the expression level of CENPF in the PCa tissues was significantly higher than that in the non-cancerous prostate tissues (IRS: PCa, 177.98 \pm 94.096 vs. benign, 121.30 $\pm 89.596 ; \mathrm{P}<0.001$ ) (Fig. 2E). Notably, during the assessment of immunostaining, we found that CENPF immunostaining occurred in the stroma between the cancer cells; however, moderate staining was observed in the benign prostate tissue (Fig. 2F and G). Furthermore, we analyzed these results from immunostaining with the limited clinical information from the TMA, but failed to find any significant association of CENPF expression with the clinicopathological characteristics of the patients with PCa (Table II).

Association of CENPF mRNA expression with the clinicopathological characteristics of the patients with PCa. Although the increased expression of CENPF in the PCa tissues was did not correlate with the clinicopathological characteristics at the protein level in our TMA cohort, we wished to analyze CENPF expression at the mRNA level. Similarly, the mRNA expression level of CENPF in the PCa tissues was significantly higher than that in the adjacent noncancerous prostate tissues at the mRNA level ( $\mathrm{PCa}, 5.67 \pm 0.47$ vs. benign, $5.40 \pm 0.11 ; \mathrm{P}<0.001)$. What is more, the Taylor dataset revealed that the increased expression of CENPF in the patients with PCa correlated with a higher GS $(\mathrm{P}=0.005)$, 

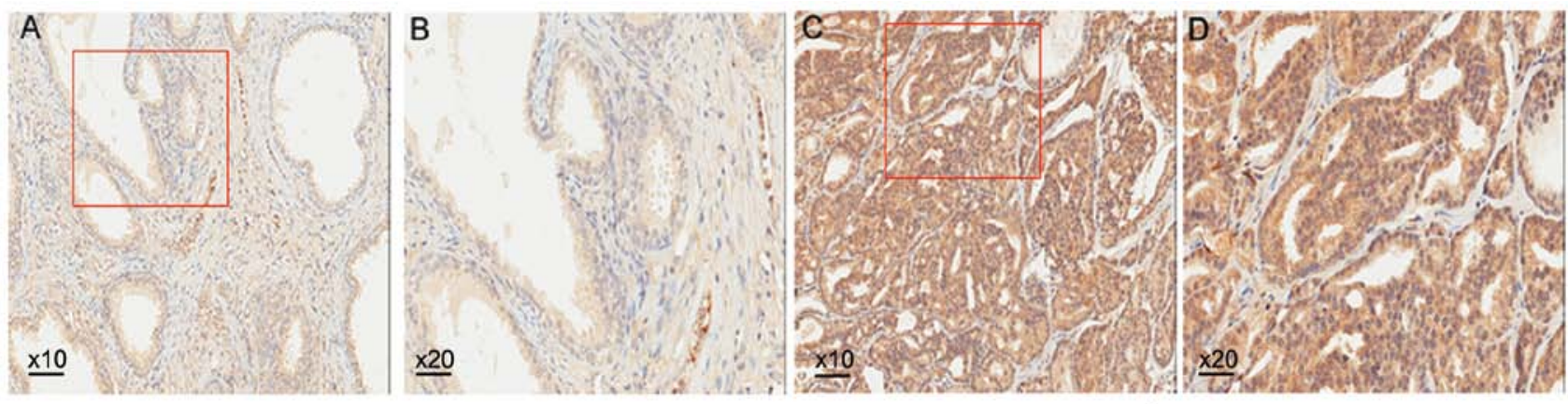

$\mathrm{E}$
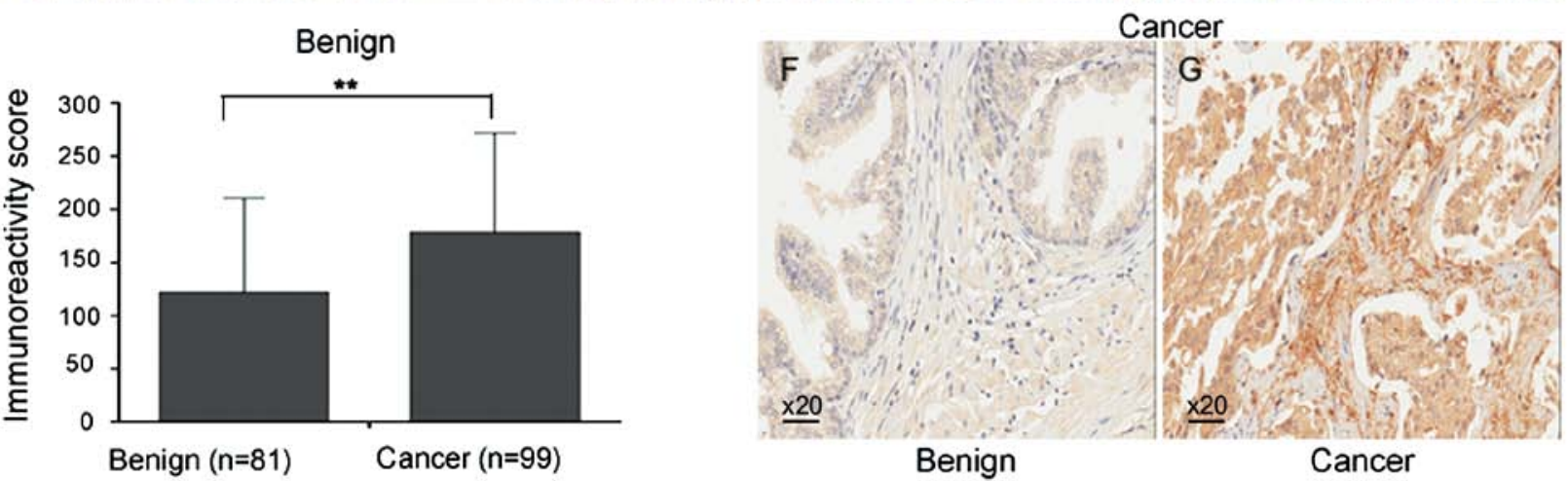

Figure 2. Immunohistochemical staining for centromere protein F (CENPF) in prostate cancer (PCa) and adjacent non-cancerous prostate tissues in our tissue microarray (TMA) samples. (A-D) Immunohistochemical staining indicated that CENPF immunostaining occurred mainly in the cytoplasm in cancer cells from PCa tissues; however, weak or moderate stianing was observed in adjacent non-cancerous prostate tissues. (E) The expression level of CENPF in PCa tissues was significantly higher than that in adjacent non-cancerous prostate tissues [immunoreactivity score (IRS): PCa, 177.98 \pm 94.096 vs. benign, 121.30 $\pm 89.596 ; \mathrm{P}<0.001$ ] ${ }^{* *} \mathrm{P}<0.01$. ( $\mathrm{F}$ and $\left.\mathrm{G}\right)$ Immunohistochemical staining of CENPF occurred in the stroma between cancer cells, but moderate staining was observed in the benign prostate tissue.
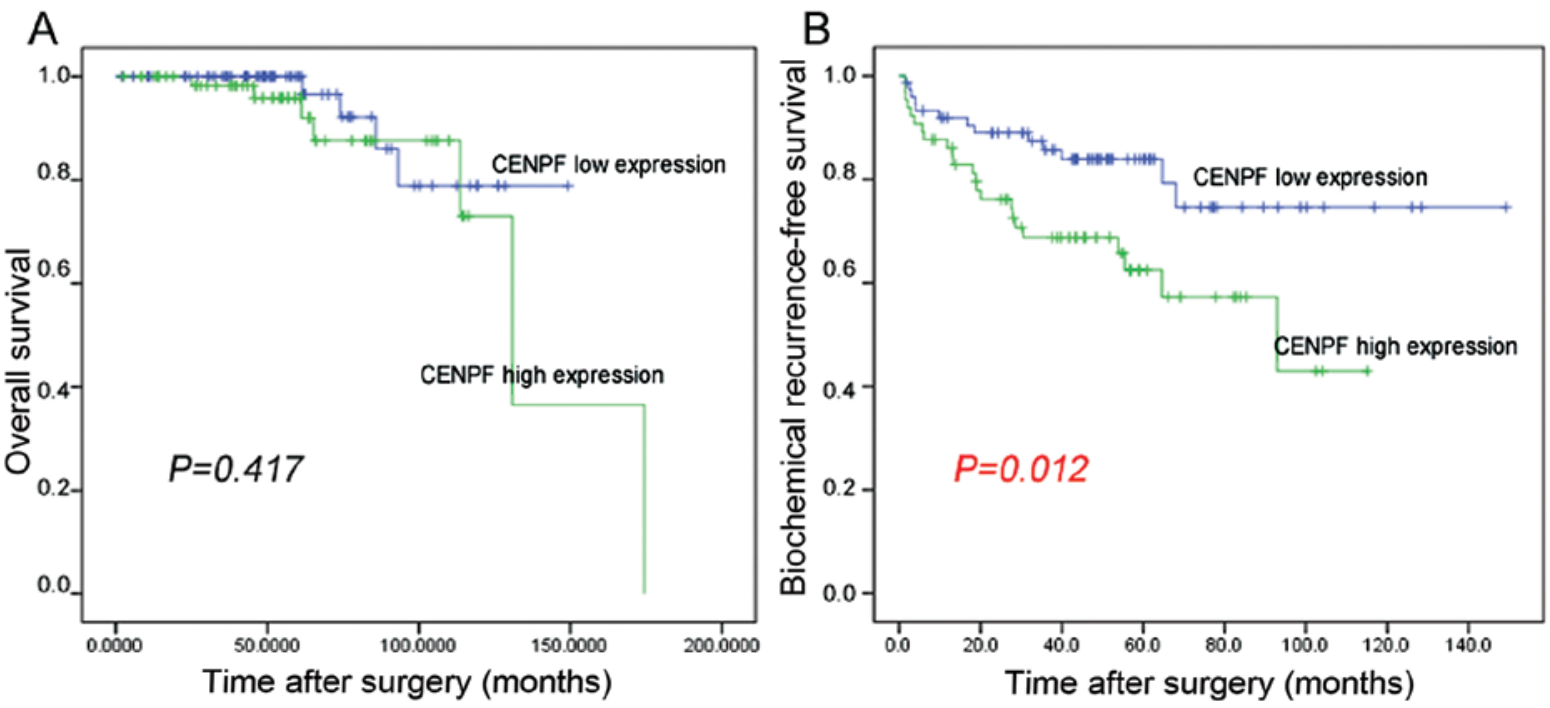

Figure 3. Kaplan-Meier survival curves of (A) overall survival and (B) biochemical recurrence (BCR)-free survival for centromere protein F (CENPF) expression in prostate cancer $(\mathrm{PCa})$.

an advanced pathological stage $(\mathrm{P}=0.008)$, the presence of metastasis $(\mathrm{P}<0.001)$, a shorter overall survival $(\mathrm{P}=0.003)$ and PSA failure $(\mathrm{P}<0.001)$ (Table II), giving us a fundamental knowledge of CENPF in clinical outcome of PCa. The expression of CENPF in the high GS group $(\mathrm{GS} \geq 8)$ was also higher than that in the intermediate GS group $(\mathrm{GS}=7)(\mathrm{P}=0.02)$ and the low GS group $(\mathrm{GS} \leq 6)(\mathrm{P}=0.013)$, although there was no statistically significant difference between the intermediate group and the low group.

Prognostic implications of CENPF expression in PCa. Using the Taylor dataset, the association of CENPF expression 
Table II. Association of CENPF expression with the clinicopathological characteristics of the patients with PCa in 2 cohorts.

\begin{tabular}{|c|c|c|c|c|c|c|}
\hline \multirow[b]{2}{*}{ Clinical characteristics } & \multicolumn{3}{|c|}{ IRS of CENPF in our cohort } & \multicolumn{3}{|c|}{ CENPF expression in Taylor dataset } \\
\hline & Cases & Mean \pm SD & P-value & Cases & Mean \pm SD & P-value \\
\hline \multicolumn{7}{|l|}{ CENPF expression } \\
\hline Benign & 81 & $121.30 \pm 89.60$ & $<0.001$ & 29 & $5.40 \pm 0.11$ & $<0.001$ \\
\hline Cancer & 99 & $177.98 \pm 94.10$ & & 150 & $5.67 \pm 0.47$ & \\
\hline \multicolumn{7}{|l|}{ Age (years) } \\
\hline$<66$ & 26 & $171.73 \pm 97.62$ & 0.695 & 125 & $5.65 \pm 0.44$ & 0.436 \\
\hline$\geq 66$ & 73 & $180.21 \pm 93.40$ & & 25 & $5.76 \pm 0.61$ & \\
\hline \multicolumn{7}{|l|}{ Serum PSA (ng/ml) } \\
\hline$<4$ & - & - & - & 24 & $5.61 \pm 0.39$ & 0.717 \\
\hline$\geq 4$ & - & - & & 123 & $5.65 \pm 0.39$ & \\
\hline \multicolumn{7}{|l|}{ Gleason score } \\
\hline$\leq 6$ & 26 & $182.88 \pm 96.20$ & 0.686 & 41 & $5.52 \pm 0.35$ & $<0.001$ \\
\hline 7 & 45 & $169.11 \pm 98.27$ & & 76 & $5.56 \pm 0.26$ & \\
\hline$\geq 8$ & 28 & $187.68 \pm 87.07$ & & 22 & $5.97 \pm 0.63$ & \\
\hline \multicolumn{7}{|l|}{ Pathological stage } \\
\hline$<\mathrm{T} 3 \mathrm{~A}$ & 70 & $176.64 \pm 95.45$ & 0.827 & 86 & $5.54 \pm 0.30$ & 0.008 \\
\hline$\geq \mathrm{T} 3 \mathrm{~A}$ & 29 & $181.21 \pm 92.33$ & & 55 & $5.74 \pm 0.49$ & \\
\hline \multicolumn{7}{|l|}{ Metastasis } \\
\hline No & 99 & $177.98 \pm 94.10$ & - & 122 & $5.54 \pm 0.28$ & $<0.001$ \\
\hline Yes & 0 & - & & 28 & $6.24 \pm 0.67$ & \\
\hline \multicolumn{7}{|l|}{ Overall survival } \\
\hline Alive & - & - & - & 131 & $5.60 \pm 0.37$ & 0.003 \\
\hline Deceased & - & - & & 19 & $6.18 \pm 0.73$ & \\
\hline \multicolumn{7}{|l|}{ PSA failure } \\
\hline Negative & - & - & - & 104 & $5.52 \pm 0.27$ & $<0.001$ \\
\hline Positive & - & - & & 36 & $5.87 \pm 0.53$ & \\
\hline
\end{tabular}

-, indicates lack of relative information of patients in our cohort. CENPF, centromere protein F; PSA, prostate-specific antigen. Taylor dataset, dataset contributed by Taylor et al (24).

with the overall survival and the BCR-free survival time of the patients with PCa were analyzed using Kaplan-Meier plots (Fig. 2). The median CENPF expression in all the PCa tissues of the Taylor dataset was used as the cut-off value to divide all the PCa tissues into high $(n=65)$ and low $(n=75)$ CENPF expression groups. As illustrated in Fig. 2, there was no statistically significant difference observed in the overall survival time between the 2 groups (Fig. 3A; $\mathrm{P}=0.417$ ); however, the BCR-free survival of the patients with $\mathrm{PCa}$ with a high CENPF expression was significantly shorter than that of the patients with a low CENPF expression (Fig. 3B; $\mathrm{P}=0.012$ ). In addition, univariate analysis revealed that there was a significant difference in the BCR-free survival [hazard ratio (HR), 3.384; 95\% confidence interval (CI), 2.066-5.541; $\mathrm{P}<0.001]$ rates between the patients with a high CENPF expression and those with a low CENPF expression. Furthermore, multivariate analysis revealed that the upregula- tion of CENPF (HR, 4.251; 95\% CI, 1.372-13.167; P=0.012), a higher GS (HR, 2.624, 95\% CI, 1.671-4.119; $\mathrm{P}<0.001)$ and a higher pre-operative PSA level (HR, 1.005; 95\% CI, 1.001-1.01; $\mathrm{P}=0.02)$ were independent predictors for a shorter BCR free-survival (Table III).

\section{Discussion}

It is known that a number of patients with localized cancer experience long-term survival following treatment with radical prostatectomy. Patients with similar clinicopathological characteristics may also have different outcomes, such as BCR and tumor metastasis. In other words, the tumor behavior of $\mathrm{PCa}$ cannot be reliably predicted by current diagnostic markers. Therefore, it is a major challenge to identify novel and effective biomarkers to provide valuable information for the early diagnosis and tumor progression of $\mathrm{PCa}$, which may help to 
Table III. Prognostic value of CENPF expression for biochemical recurrence-free survival by Cox proportional hazards model.

Biochemical recurrence-free survival

Hazard ratio $(95 \% \mathrm{CI}) \quad$ P-value

\begin{tabular}{llc}
\hline Univariate analysis & & \\
CENPF & $3.384(2.066-5.541)$ & $<0.001$ \\
Gleason score & $3.367(2.39-4.743)$ & $<0.001$ \\
Pre-operative PSA & $1.005(1.001-1.008)$ & 0.01 \\
Pathological stage & $5.232(2.564-10.676)$ & $<0.001$ \\
Age & $1.019(0.972-1.069)$ & 0.434 \\
Clinical stage & $1.032(0.53-2.011)$ & 0.926 \\
Multivariate analysis & & \\
CENPF & $4.251(1.372-13.167)$ & 0.012 \\
Gleason score & $2.624(1.671-4.119)$ & $<0.001$ \\
Pre-operative PSA & $1.005(1.001-1.01)$ & 0.02 \\
Pathological stage & $2.389(0.979-5.831)$ & 0.056 \\
Age & $0.993(0.942-1.047)$ & 0.806 \\
Clinical stage & $1.151(0.505-2.627)$ & 0.737 \\
\end{tabular}

CENPF, centromere protein $\mathrm{F}$.

establish personalized treatment and lessen the morbidity for patients with PCa.

The present study suggests that CENPF is an oncogene which plays an important role in tumor progression in $\mathrm{PCa}$. More importantly, the increased expression of CENPF may efficiently predict BCR-free survival in patients with PCa.

It has been demonstrated that CENPF is upregulated in primary breast cancer (25). As for hepatocellular carcinoma (HCC), CENPF has been found to be overexpressed in tumor tissue compared with non-tumor tissue (26). Silencing CENPF can decrease the ability of HCC cells to proliferate, form colonies and induce tumor formation in nude mice (18). In the present study, using clinical samples, we also found that the expression of CENPF in PCa tissues was significantly higher than that in non-cancerous prostate tissues, which was consistent with the results of previous studies on other tumor types (17-23). However, we failed to find any correlation of CENPF with the clinicopathological characteristics of the patients with PCa with limited information. By immunostaining of samples from a TMA which contained primary tumor and PCa metastatic samples, Aytes et al (27) similarly found that only the increased expression of FOXM1 or CENPF at the protein level was not predictive of an unfavorable outcome, even though the co-expression of FOXM1 and CENPF was associated with a worse outcome in $\mathrm{PCa}$. These results indicate that CENPF may be closely linked to cell proliferation in $\mathrm{PCa}$. The overexpression of CENPF in PCa tissues may be a consequence of malignant transformation or may play a modulatory role in abnormal cell division.

On the other hand, a higher CENPF expression has been previously reported to correlate with the clinical stage, $T$ classification and a shorter overall survival in esophageal squamous cell carcinoma (28). DNA microarrays and TMAs have also revealed that the upregulation of CENPF is significantly associated with a high tumor grade, a worse overall survival and a reduced metastasis-free survival in patients with primary breast cancer $(17,25)$. Our present data indicated that the upregulation of CENPF mRNA expression was associated with a higher GS, an advanced pathological stage, the presence of metastasis, a shorter overall survival and PSA failure. Furthermore, when Kaplan-Meier plots and the Cox proportional hazards model were used to evaluate the association of CENPF expression with the outcome of surgical treatment in the Taylor dataset, statistical analysis revealed that the increased expression of CENPF correlated with a shorter BCR-free survival. In addition, CENPF expression, GS and the pre-operative PSA level were independent predictors of BCR-free survival, as shown by multivariate analysis.

Further analyses with sufficient clinical information are required in order to better understand the different correlations of CENPF protein and mRNA expression with the clinicopathological characteristics of patients with $\mathrm{PCa}$, which is a heterogeneous multifocal disease.

Although CENPF is initially known for its association with the centromere-kinetochore complex and chromosomal segregation, previous studies have demonstrated that CENPF may play a role in the regulation of the mitosis and proliferation of malignant cell growth, which are mediated by protein interactions (13,29-32). Thus, the actual functional role of CENPF is more elusive and complex. However, the mRNA expression or other genetic variations in the CENPF gene are likely to represent a fundamental aspect of PCa biology and may prove to be useful in the prognosis of the disease in clinical practice. On the other hand, CENPF is one of the genes related to chromosomal instability (CIN) and CCP. CIN, in multiple human cancers, is considered to contribute to tumourigenesis, tumor development and progression through its involvement in the amplification of oncogenes, the loss of heterozygosity of tumor suppressor genes, increasing cell proliferation and decreasing cell death (33). The CCP score, as discussed above, has recently been shown to be strongly predictive of clinical outcome in patients with PCa (10-12), bladder and lung adenocarcinoma (34) from different cohorts.

In conclusion, the findings from the present study extend our knowledge of the oncogene and prognostic significance of CENPF in PCa. The results from our study not only provide new insight into the progression of $\mathrm{PCa}$, but also provide useful information which may aid clinicians to design more personalized treatment for patients with $\mathrm{PCa}$.

\section{Acknowledgements}

The present study was supported by grants from the National Natural Science Foundation of China (81170699, 81272813), the Guangdong Provincial Science and Technology Project (2012B031800008), Guangzhou Municipal Science and the Technology Key Project (11C23150711).

\section{References}

1. Jemal A, Bray F, Center MM, et al: Global cancer statistics. CA Cancer J Clin 61: 69-90, 2011. 
2. Siegel R, Ma J, Zou Z and Jemal A: Cancer statistics, 2014. CA Cancer J Clin 64: 9-29, 2014.

3. Center MM, Jemal A, Lortet-Tieulent J, et al: International variation in prostate cancer incidence and mortality rates. Eur Urol 61: 1079-1079, 2012.

4. Utomo NB, Mochtar CA and Umbas R: Primary hormonal treatment in localized and locally advanced prostate cancer: effectiveness and survival predictive factors. Acta Med Indones 44: 10-15, 2012.

5. Ferlay J, Parkin DM and Steliarova-Foucher E: Estimates of cancer incidence and mortality in Europe in 2008. Eur J Cancer 46: 765-781, 2010.

6. Molitierno J, Evans A, Mohler JL, et al: Characterization of biochemical recurrence after radical prostatectomy. Urol Int 77: 130-134, 2006

7. Stephenson AJ, Scardino PT, Eastham JA, et al: Postoperative nomogram predicting the 10 -year probability of prostate cancer recurrence after radical prostatectomy. J Clin Oncol 23 7005-7012, 2005.

8. Shariat SF, Karakiewicz PI, Roehrborn CG and Kattan MW: An updated catalog of prostate cancer predictive tools. Cancer 113: 3075-3099, 2008.

9. Alers JC, Rochat J, Krijtenburg PJ, Hop WC, Kranse R, Rosenberg C, Tanke HJ, Schröder FH and van Dekken H: Identification of genetic markers for prostatic cancer progression. Lab Invest 80: 931-942, 2000.

10. Cuzick J, Swanson GP, Fisher G, et al: Prognostic value of an RNA expression signature derived from cell cycle proliferation genes in patients with prostate cancer: a retrospective study. Lancet Oncol 12: 245-255, 2011.

11. Arsov C, Jankowiak F, Hiester A, Rabenalt R, Quentin M, Schimmller L, Blondin D, Antoch G and Albers P: Prognostic value of a cell-cycle progression score in men with prostate cancer managed with active surveillance after MRI-guided prostate biopsy - a pilot study. Anticancer Res 34: 2459-2466, 2014.

12. Cooperberg MR, Simko JP, Cowan JE, et al: Validation of a cellcycle progression gene panel to improve risk stratification in a contemporary prostatectomy cohort. J Clin Oncol 31: 1428-1434, 2013.

13. Varis A, Salmela AL and Kallio MJ: Cenp-F (mitosin) is more than a mitotic marker. Chromosoma 115: 288-295, 2006.

14. Liao H, Winkfein RJ, Mack G, Rattner JB and Yen TJ: CENP-F is a protein of the nuclear matrix that assembles onto kinetochores at late G2 and is rapidly degraded after mitosis. J Cell Biol 130 507-518, 1995.

15. Rattner JB, Rao A, Fritzler MJ, Valencia DW and Yen TJ: CENP-F is a ca $400 \mathrm{kDa}$ kinetochore protein that exhibits a cell-cycle dependent localization. Cell Motil Cytoskeleton 26: 214-226, 1993

16. Hussein D and Taylor SS: Farnesylation of Cenp-F is required for G2/M progression and degradation after mitosis. J Cell Sci 115: 3403-3414, 2002.

17. Brendle A, Brandt A, Johansson R, Enquist K, Hallmans G, Hemminki K, Lenner P and Forsti A: Single nucleotide polymorphisms in chromosomal instability genes and risk and clinical outcome of breast cancer: a Swedish prospective case-control study. Eur J Cancer 45: 435-442, 2009.

18. Dai Y, Liu L, Zeng T, Zhu YH, Li J, Chen L, Li Y, Yuan YF, Ma S and Guan XY: Characterization of the oncogenic function of centromere protein $\mathrm{F}$ in hepatocellular carcinoma. Biochem Biophys Res Commun 436: 711-718, 2013.
19. Chen WB, Cheng XB, Ding W, Wang YJ, Chen D, Wang JH and Fei RS: Centromere protein $\mathrm{F}$ and survivin are associated with high risk and a poor prognosis in colorectal gastrointestinal stromal tumours. J Clin Pathol 64: 751-755, 2011.

20. Cao JY, Liu L, Chen SP, et al: Prognostic significance and therapeutic implications of centromere protein $\mathrm{F}$ expression in human nasopharyngeal carcinoma. Mol Cancer 9: 237, 2010.

21. Erlanson M, Casiano CA, Tan EM, Lindh J, Roos G and Landberg G: Immunohistochemical analysis of the proliferation associated nuclear antigen CENP-F in non-Hodgkin's lymphoma. Mod Pathol 12: 69-74, 1999.

22. Shigeishi H, Mizuta K, Higashikawa K, Yoneda S, Ono S and Kamata N: Correlation of CENP-F gene expression with tumorproliferating activity in human salivary gland tumors. Oral Oncol 41: 716-722, 2005.

23. Albino D, Scaruffi P, Moretti S, Coco S, Truini M, Di Cristofano C, Cavazzana A, Stigliani S, Bonassi S and Tonini GP: Identification of low intratumoral gene expression heterogeneity in neuroblastic tumors by genome-wide expression analysis and game theory. Cancer 113: 1412-1422, 2008.

24. Taylor BS, Schultz N, Hieronymus H, et al: Integrative genomic profiling of human prostate cancer. Cancer Cell 18: 11-22, 2010.

25. O'Brien S L, Fagan A, Fox E J, et al: CENP-F expression is associated with poor prognosis and chromosomal instability in patients with primary breast cancer. Int J Cancer 120: 1434-1443, 2007.

26. Kim HE, Kim DG, Lee KJ, et al: Frequent amplification of CENPF, GMNN and CDK13 genes in hepatocellular carcinomas. PLoS One 7: e43223, 2012.

27. Aytes A, Mitrofanova A, Lefebvre C, et al: Cross-species regulatory network analysis identifies a synergistic interaction between FOXM1 and CENPF that drives prostate cancer malignancy. Cancer Cell 25: 638-651, 2014.

28. Mi YJ, Gao J, Xie JD, et al: Prognostic relevance and therapeutic implications of centromere protein $\mathrm{F}$ expression in patients with esophageal squamous cell carcinoma. Dis Esophagus 26: 636-643, 2013

29. Bomont P, Maddox P, Shah JV, Desai AB and Cleveland DW: Unstable microtubule capture at kinetochores depleted of the centromere-associated protein CENP-F. EMBO J: 24: 3927-3939, 2005.

30. Feng J, Huang $\mathrm{H}$ and Yen TJ: CENP-F is a novel microtubulebinding protein that is essential for kinetochore attachments and affects the duration of the mitotic checkpoint delay. Chromosoma 115: 320-329, 2006.

31. Holt SV, Vergnolle MA, Hussein D, Wozniak MJ, Allan VJ and Taylor SS: Silencing Cenp-F weakens centromeric cohesion, prevents chromosome alignment and activates the spindle checkpoint. J Cell Sci 118: 4889-4900, 2005.

32. Ma L, Zhao X and Zhu X: Mitosin/CENP-F in mitosis, transcriptional control, and differentiation. J Biomed Sci 13: 205-213, 2006.

33. Jefford CE and Irminger-Finger I: Mechanisms of chromosome instability in cancers. Crit Rev Oncol Hematol 59: 1-14, 2006.

34. Dancik GM and Theodorescu D: Robust prognostic gene expression signatures in bladder cancer and lung adenocarcinoma depend on cell cycle related genes. PLoS One 9: e85249, 2014. 